Pacific Journal of Mathematics

THE REGULAR REPRESENTATION OF LOCAL AFFINE 


\title{
THE REGULAR REPRESENTATION OF LOCAL AFFINE MOTION GROUPS
}

\author{
BRUCE E. BLACKADAR
}

Let $F$ be a nondiscrete locally compact topological field. Then the regular representation of the group of invertible affine motions of $F^{n}$, the semidirect product of $F^{n}$ by $G L_{n}(F)$, is a type $I_{\infty}$ factor. An explicit transformation formula is obtained.

1. Introduction. It is of some interest [4] to examine the regular representation of the group of affine motions of $F^{n}$ for a nondiscrete locally compact field $F$. We show that the regular representation of such a group is a type $I_{\infty}$ factor, i.e. is a multiple of an irreducible representation on an infinite-dimensional Hilbert space.

The results of this paper were part of the author's doctoral dissertation at the University of California, Berkeley, June 1975, under the direction of Calvin C. Moore.

2. Preliminaries. Let $F$ be a nondiscrete locally compact field. It is known (see, for example, [3, Theorem 9.21]) that $F$ is either $\mathbf{R}, \mathbf{C}$, a finite extension of the field $\mathbf{Q}_{p}$ of $p$-adic numbers, or the field of formal Laurent series in one variable over a finite field. In particular, if $F$ is not $\mathbf{R}$ or $\mathbf{C}$ it has the following properties:

(i) $F$ is the quotient field of a compact open subring $R$.

(ii) $R$ has a unique maximal ideal $M$, which is principal; let $M=(\pi)$.

(iii) $R / M$ is a finite field with (say) $q$ elements.

(iv) There is a character $\chi$ on the additive group of $F$ with $R \subseteq \operatorname{ker} \chi$, $\pi^{-1} \notin$ ker $\chi$; any other character on $F$ is of the form $\chi_{u}(x)=\chi(u x)$ for some $u \in F$.

(v) $R$ has a nonarchimedean absolute value $|\cdot|$ with $|\pi|=1 / q$.

(vi) If $\mu$ (usually denoted $d x$ ) is additive Haar measure on $F$, normalized so that $\mu(R)=1$, then $\mu(M)=1 / q$ and $d x /|x|$ is multiplicative Haar measure $\mu^{*}$ on $F^{*}$, with the measure of $R^{*}$ equal to $1-1 / q$.

If $F$ is $\mathbf{R}$ or $\mathbf{C}$, let $d x$ denote Lebesgue measure normalized to make the Fourier inversion formula valid, $|\cdot|$ the ordinary absolute value (squared if $F=\mathbf{C}$ ), and $\chi(x)=e^{2 \pi i \operatorname{Re} x}$. 
We now let $G_{n}$ be the group of invertible affine motions of $F^{n}$ (the $n$-dimensional " $a x+b$ " group), i.e. $G_{n}=F^{n} \cdot G L_{n}$, the semidirect product of $F^{n}$ by $G L_{n}=G L_{n}(F)$. It will frequently be useful to consider $G_{n}$ as a subgroup of $G L_{n+1}$ by the identification

$$
(b, A) \leftrightarrow\left[\begin{array}{cccc}
1 & 0 & \cdots & 0 \\
b & & A &
\end{array}\right] .
$$

Using this identification, we will think of $G_{1} \subseteq G L_{2} \subseteq \cdots \subseteq G L_{n} \subseteq G_{n}$ $\subseteq G L_{n+1}$.

\section{The results.}

THEOREM 3.1. The right regular representation $\rho_{G_{n}}$ of $G_{n}$ is a type $I_{\infty}$ factor.

Proof. By induction on $n$. The case $n=1$ was done in [2, §3]; we briefly outline the argument for completeness. $G_{1} \cong F \times F^{*}$ topologically, and $\mu \times \mu^{*}$ is right Haar measure. If $f \in L^{2}\left(G_{1}\right)$, set $\hat{f}_{u}(y, x)=$ $\chi(u y) \int_{F} f(z, x) \chi(-u z) d z$; then $\left[\rho_{G_{1}}(b, a) f\right]_{u}(y, x)=\chi(u b x) \hat{f_{u}}(y, a x)$. If $\rho_{u}=$ ind $_{F \uparrow G_{1}} \chi_{-u}$, then $\rho_{u} \cong \rho_{v}$ for $u, v \neq 0$; since $f(y, x)=\int_{F} \hat{f}_{u}(y, x) d u$, we have $\rho=\int_{F} \rho_{u} d u$.

Now assume $\rho_{G_{n-1}}$ is a factor. Regard $F^{n}$ as a subgroup of $G_{n}$ by identifying $b$ with $(b, \mathbf{1}) \cdot \rho_{G_{n}}=\operatorname{ind}_{F^{n} \uparrow G_{n}} \rho_{F^{n}} . \rho_{F^{n}}=\int_{F^{n}} \chi_{u} d u$, where $\chi_{u}$ $\left(u \in F^{n}\right)$ is the character given by $\chi_{u}(v)=\chi(u \cdot v)$. By moving the direct integral past the induction, we get $\rho_{G_{n}}=\int_{F^{n}}\left(\operatorname{ind}_{F^{n} \uparrow G_{n}} \chi_{u}\right) d u$. If $u$ and $v$ are nonzero vectors in $F^{n}$, ind $\chi_{u} \simeq$ ind $\chi_{v}$, since $u$ and $v$ are conjugate under the action of $G L_{n}$ on $F^{n}$. Set $e_{1}=(1,0, \ldots, 0)$. We then have $\rho_{G_{n}} \simeq$ $\int_{F^{n}}\left(\right.$ ind $\left._{F^{n} \uparrow G_{n}} \chi_{e_{1}}\right) d u$. $G_{n}=F^{n} \cdot G L_{n}$, so, regarding $G_{n-1} \subseteq G L_{n}$, let $H_{n}=$ $F^{n} \cdot G_{n-1}$. Since the action of $G_{n-1}$ on $F^{n}$ leaves the first coordinate fixed, we have $H_{n}=F \times\left(F^{n-1} \cdot G_{n-1}\right)$.

We split the induction into two steps,

$$
\rho_{G_{n}} \simeq \int_{F^{n}} \operatorname{ind}_{H_{n} \uparrow G_{n}}\left(\text { ind }_{F^{n} \uparrow H_{n}} \chi_{e_{1}}\right) d u .
$$

Let us examine $\pi=\operatorname{ind}_{F^{n} \uparrow H_{n}} \chi_{e_{1}} \cdot \chi_{e_{1}}=\chi \otimes 1$ on $F^{n}=F \times F^{n-1}$, and $H_{n}=F \times\left(F^{n-1} \cdot G_{n-1}\right)$, so $\pi \simeq \chi \otimes\left(\operatorname{ind}_{F^{n-1} \uparrow\left(F^{n-1} \cdot G_{n-1}\right)} 1\right) \simeq \chi \otimes \rho_{G_{n-1}}$ (where $\rho_{G_{n-1}}$ is considered as a representation of $F^{n-1} \cdot G_{n-1}$ with kernel $\left.F^{n-1}\right)$. By the induction hypothesis, $\rho_{G_{n-1}}$ is a $I_{\infty}$ factor representation of $G_{n-1}$, so $\pi$ is a $I_{\infty}$ factor representation of $H_{n}$. We now use Mackey's theorem ([1], Theorem 6, p. 58) to show that $\operatorname{ind}_{H_{n} \uparrow G_{n}} \pi$ is a $I_{\infty}$ factor representation of $G_{n}$, since $H_{n}$ is precisely the stability group of $\chi_{e_{1}}$ under the action of $G_{n}$ on $F^{n}$. 
We now get an explicit formula for this transformation. Throughout, we will always consider $G L_{k} \subseteq G_{k} \subseteq G L_{k+1} \subseteq G_{k+1}$, so that all groups will be thought of as being embedded in $G L_{n+1}$. Let $f \in L^{2}\left(G_{n}\right)$. We first take the Fourier transform along $F^{n}$ : define

$$
\hat{f}_{u}(y, X)=\chi(u \cdot y) \int_{F^{n}} f(z, X) \chi(-u \cdot z) d z .
$$

Then

$$
\begin{aligned}
\hat{f}_{u} \in \mathcal{K}_{u}^{n}=\left\{f: G_{n} \rightarrow \mathbf{C}: f(y, X)=\right. & \chi(u \cdot y) f(0, X), \\
& \left.\int_{G L_{n}}|f(0, X)|^{2} d X<\infty\right\}
\end{aligned}
$$

where $d X$ is Haar measure on $G L_{n}$.

By the Fourier inversion formula, $f(y, X)=\int_{F^{n}} \hat{f}_{u}(y, X) d u$.

$$
\begin{aligned}
{[\rho(b, A) f]_{u}^{\wedge}(y, X) } & =\chi(u \cdot y) \int_{F^{n}}[\rho(b, A) f](z, X) \chi(-u \cdot z) d z \\
& =\chi(u \cdot y) \int_{F^{n}} f(z+X b, X A) \chi(-u \cdot z) d z
\end{aligned}
$$

Set $t=z-X b$.

$$
\begin{aligned}
& =\chi(u \cdot y) \int_{F^{n}} f(t, X A) \chi(-u \cdot t) \chi(u \cdot X b) d t \\
& =\chi(u \cdot X b) \hat{f}_{u}(y, X A) .
\end{aligned}
$$

This is precisely the representation $\operatorname{ind}_{F^{n} \uparrow G_{n}} \chi_{u}$ on $\mathcal{H}_{u}^{n}\left[\chi_{u}(v)=\chi(u \cdot v)\right]$. So we have written

$$
L^{2}\left(G_{n}\right) \simeq \int_{F^{n}} \mathcal{K}_{u}^{n} d u, \quad \rho_{G_{n}} \simeq \int_{F^{n}}\left(\operatorname{ind}_{F^{n} \uparrow G_{n}} \chi_{u}\right) d u .
$$

Let $e_{1}^{n}=(1,0, \ldots, 0) \in F^{n}$. We now take an equivalence in each piece, $\mathcal{H}_{u}^{n} \rightarrow \mathcal{H}_{e_{1}^{n}}^{n}$, ind $\chi_{u} \rightarrow$ ind $\chi_{e_{1}^{n}}$ by setting $\tilde{f}_{u}(y, X)=\hat{f}_{u}\left(B_{u}(y, X)\right)$ where

$$
B_{u}=\left[\begin{array}{cccc}
1 / u_{1} & -u_{2} / u_{1} & \cdots & -u_{n} / u_{1} \\
0 & 1 & \cdots & 0 \\
\vdots & \vdots & \ddots & \vdots \\
0 & 0 & \cdots & 1
\end{array}\right] \text { for } u=\left(u_{1}, \ldots, u_{n}\right), u_{1} \neq 0
$$

We interchangeably think of $B_{u}$ as an element of $G L_{n}, G_{n}$, and $G L_{n+1}$ to simplify notation. The reason for choosing this $B_{u}$ is that $u \cdot B_{u} v=$ $B_{u}^{t} u \cdot v=e_{1}^{n} \cdot v$ for all $v$. 
$\hat{f}_{u} \rightarrow \tilde{f}_{u}$ is an isometry of $\mathcal{H}_{u}^{n}$ onto $\mathcal{H}_{e_{1}^{n}}^{n}$ : this can be seen most easily by identifying $\mathcal{K}_{u}^{n}$ with $L^{2}\left(G L_{n}\right)$ by $\hat{f}_{n} \leftrightarrow \hat{f}_{u}(0, \cdot)$ and noting that $G L_{n}$ is unimodular (we have assumed right Haar measure). By associating $f$ with $\int_{F^{n}} \tilde{f}_{u} d u$, we get

$$
L^{2}\left(G_{n}\right) \simeq \int_{F^{n}} \mathcal{H}_{e_{1}^{n}}^{n} d u, \quad \rho_{G_{n}} \simeq \int_{F^{n}}\left(\operatorname{ind}_{F^{n} \uparrow G_{n}} \chi_{e_{1}^{n}}\right) d u .
$$

$\tilde{f}_{u}(y, X)=\chi\left(e_{1}^{n} \cdot y\right) \int_{F^{n}} f\left(v, B_{u} X\right) \chi(-u \cdot v) d v$. We now change variables, setting $v=B_{u} t, d v=1 /\left|u_{1}\right| d t$.

$$
\begin{aligned}
\tilde{f}_{u}(y, X) & =\chi\left(e_{1}^{n} \cdot y\right) \int_{F^{n}} f\left(B_{u}(t, X)\right) \chi\left(-u \cdot B_{u} t\right) \frac{1}{\left|u_{1}\right|} d t \\
& =\chi\left(e_{1}^{n} \cdot y\right) \int_{F^{n}} f\left(B_{u}(t, X)\right) \chi\left(e_{1}^{n} \cdot t\right) d t .
\end{aligned}
$$

Now we split the induction into two steps,

$$
\operatorname{ind}_{F^{n} \rightarrow G_{n}} \chi_{e_{1}^{n}}=\operatorname{ind}_{H_{n} \uparrow G_{n}}\left(\operatorname{ind}_{F^{n} \uparrow H_{n}} \chi_{e_{1}^{n}}\right) \text {. }
$$

Set

$$
\begin{gathered}
\bar{f}_{u}(y, X)(Z)=\tilde{f}_{u}(y, Z X) \quad \text { for } y \in F^{n}, X \in G L_{n}, Z \in G_{n-1} \subseteq G L_{n} . \\
\bar{f}_{u} \in\left\{f: G_{n} \rightarrow L^{2}\left(G_{n-1}\right): f([(b, C)(y, X)])(Z)=\chi\left(e_{1}^{n} \cdot b\right) f(y, X)(Z C)\right. \\
\text { for } \left.X \in G L_{n}, Z, C \in G_{n-1}, b, y \in F^{n} ; \int_{G L_{n}}|f(X)(\mathbf{1})|^{2} d X<\infty\right\} .
\end{gathered}
$$

If we look at the representation $\sigma^{n}$ of $H_{n}$ on $L^{2}\left(G_{n-1}\right)$ given by $\left[\sigma^{n}(b, C) g\right](Z)=\chi\left(e_{1}^{n} \cdot b\right) g(Z C)$ for $b \in F^{n}, C \in G_{n-1}$, we see that

$$
\sigma^{n} \simeq \operatorname{ind}_{F^{n} \uparrow H_{n}} \chi_{e_{1}^{n}}, \text { and } \operatorname{ind}_{F^{n} \uparrow G_{n}} \chi_{e_{1}^{n}} \simeq \operatorname{ind}_{H_{n} \uparrow G_{n}} \sigma^{n} .
$$

Also, $\sigma^{n} \simeq \chi_{e_{1}^{n}} \otimes \rho_{G_{n-1}}$ as an inner tensor product.

We now decompose $\rho_{G_{n-1}}$ in the same manner as before. Let

$$
\begin{aligned}
\hat{f}_{u, r}(y, X)(t, S)=\chi(r \cdot t) \int_{F^{n-1}} \bar{f}_{u}(y, X)(w, S) \chi(-r \cdot w) d w & \\
& \left(t \in F^{n-1}, S \in G L_{n-1}\right) .
\end{aligned}
$$

Then

$$
\bar{f}_{u}(y, X)(t, S)=\int_{F^{n-1}} \hat{f}_{u, r}(y, X)(t, S) d r ; \quad \hat{f}_{u, r}(y, X) \in \mathcal{H}_{r}^{n-1}
$$


Let

$$
B_{r}=\left[\begin{array}{cccc}
1 / r_{1} & -r_{2} / r_{1} & \cdots & -r_{n-1} / r_{1} \\
0 & 1 & \cdots & 0 \\
\vdots & \vdots & \ddots & \vdots \\
0 & 0 & \cdots & 1
\end{array}\right] \in G L_{n-1}
$$

$$
\text { (for } r \in F^{n-1}, r_{1} \neq 0 \text { ). }
$$

Set $\tilde{f}_{u, r}(y, X)(t, S)=\hat{f}_{u, r}(y, X)\left(B_{r}(t, S)\right)$.

$$
\begin{aligned}
& {\left[\sigma^{n}(b,(d, C)) f\right]_{u, r}^{\wedge}(y, X)(t, S)} \\
& \quad=\chi(r \cdot t) \int_{F^{n-1}}\left[\sigma^{n}(b,(d, C)) f\right]_{u}^{\tilde{y}}(y, X)(w, S) \chi(-r \cdot w) d w \\
& \quad=\chi(r \cdot t) \int_{F^{n-1}} \chi\left(e_{1} \cdot b\right) \tilde{f}_{u}(y, X)(w+S d, S C) \chi(-r \cdot w) d w .
\end{aligned}
$$

Set $v=w+S d$.

$$
\begin{aligned}
& =\chi\left(e_{1} \cdot b\right) \chi(r \cdot t) \int_{F^{n-1}} \tilde{f}_{u}(y, X)(v, S C) \chi(-r \cdot v) \chi(r \cdot S d) d v \\
& =\chi\left(e_{1} \cdot b\right) \chi(r \cdot S d) \hat{f}_{u, r}(y, X)(t, S C) \\
& {\left[\sigma^{n}(b,(d, C)) f\right]_{u, r}^{\sim}(y, X)(t, S)} \\
& =\chi\left(e_{1} \cdot b\right) \chi\left(r \cdot B_{r} S d\right) \hat{f}_{u, r}(y, X)\left(B_{r}(t, S C)\right) \\
& =\chi\left(e_{1} \cdot b\right) \chi\left(e_{1} \cdot S d\right) \tilde{f}_{u, r}(y, X)(t, S C) .
\end{aligned}
$$

Thus by associating $\tilde{f}_{u}$ with

$$
\begin{gathered}
\int_{F^{n-1}} \tilde{f}_{u, r} d r, \quad \sigma^{n} \simeq \int_{F^{n-1}} \chi_{e_{1}^{n}} \otimes\left(\operatorname{ind}_{F^{n-1} \uparrow G_{n-1}} \chi_{e_{1}^{n-1}}\right) . \\
\tilde{f}_{u, r}(y, X)(t, S)=\chi\left(e_{1} \cdot t\right) \int_{F^{n-1}} \tilde{f}_{u}(y, X)\left(w, B_{r} S\right) \chi(-r \cdot w) d w .
\end{gathered}
$$

We want to pull the $B_{r}$ past the $w$, so we change variables as before. Set $w=B_{r} v, d w=1 /\left|r_{1}\right| d v$. Then

$$
\begin{aligned}
\tilde{f}_{u, r}(y, X)(t, S)=\chi\left(e_{1} \cdot t\right) \int_{F^{n-1}} \tilde{f}_{u}(y, X)\left(B_{r}(v, S)\right) \chi\left(-r \cdot B_{r} v\right) \frac{1}{\left|r_{1}\right|} d v \\
=\chi\left(e_{1} \cdot t\right) \int_{F^{n-1}} \tilde{f}_{u}(y, X)\left(B_{r}(v, S)\right) \chi\left(e_{1} \cdot v\right) \frac{1}{\left|r_{1}\right|} d v \\
=\chi\left(e_{1}^{n} \cdot y\right) \chi\left(e_{1}^{n-1} \cdot t\right) \\
\quad \cdot \int_{F^{n-1}}\left[\int_{F^{n}} f\left(B_{u}\left(w, B_{r}(v, S) X\right)\right) \chi\left(-w_{1}\right) \frac{1}{\left|u_{1}\right|} d w\right] \chi\left(-v_{1}\right) \frac{1}{\left|r_{1}\right|} d v
\end{aligned}
$$


We now pull the $B_{r}$ past the $w$, by letting $w=B_{r} z, d w=1 /\left|r_{1}\right| d z$. Note that $z_{1}=w_{1}$ since $B_{r}$ does not affect the first column.

$$
\begin{aligned}
& \tilde{f}_{u, r}(y, X)(t, S) \\
& \quad=\int_{F^{n-1}}\left[\int_{F^{n}} f\left(B_{u} B_{r}(z,(v, S) X)\right) \chi\left(-z_{1}\right) \frac{1}{\left|u_{1} r_{1}\right|} d z\right] \chi\left(-v_{1}\right) \frac{1}{\left|r_{1}\right|} d v .
\end{aligned}
$$

We now repeat the process until we get down to $F^{1}$. We end up with

$$
\begin{gathered}
\tilde{f}_{u, r, \ldots, s}(y, X)(t, S) \cdots(q, T) \\
\left((y, X) \in G_{n},(t, S) \in G_{n-1}, \ldots,(q, T) \in G_{1}\right) \\
=\chi\left(e_{1}^{n} \cdot y\right) \chi\left(e_{1}^{n-1} \cdot t\right) \cdots \chi(q) \\
\quad \cdot \int_{F} \int_{F^{2}} \cdots \int_{F^{n}} f\left(B_{u} B_{r} \cdots B_{s}(w,(v, \ldots(z, T) \ldots, S) X)\right) \\
\cdot \chi\left(-w_{1}-v_{1}-\cdots-z_{1}\right) \frac{1}{\left|u_{1} r_{1}^{2} \cdots s_{1}^{n}\right|} d w d v \cdots d z . \\
\tilde{f}_{u, r, \ldots, s} \in \mathcal{F C}^{n}=\left\{f: G_{n} \rightarrow \mathcal{H}^{n-1}: f([(b, C)(y, X)])(Z)\right. \\
=\chi\left(e_{1}^{n} \cdot b\right) f(y, X)(Z C) \quad \text { for } X \in G L_{n}, Z, C \in G_{n}, \\
\left.b, y \in F^{n} ; \int_{G_{n-1} \backslash G_{n}}|f(y, X)|^{2}<\infty\right\} .
\end{gathered}
$$

$\left[\mathcal{H}^{0}=\mathbf{C}\right]$.

$\operatorname{Set} \bar{f}_{u, r, \ldots, s}(y, X)=\tilde{f}_{u, r, \ldots, s}(y, X)(0, \mathbf{1}) \cdots(0, \mathbf{1})$.

$\bar{f}_{u, r, \ldots, s} \in \mathcal{H}=\left\{f: G_{n} \rightarrow \mathbf{C}: f(C(y, X))=\phi(C) f(y, X)\right.$

$$
\text { for } \left.C \in \Gamma_{n}, \int_{\Gamma_{n} \backslash G_{n}}|f(y, X)|^{2}<\infty\right\}
$$

where

$$
\Gamma_{n}=\left\{\left[\begin{array}{cccc}
1 & & & \\
& 1 & & \\
& & \ddots & \\
* & & & 1
\end{array}\right]\right\}, \quad \phi\left(\left[\begin{array}{ccccc}
1 & & & \\
a_{11} & 1 & & 0 & \\
\vdots & & \ddots & \\
a_{n 1} & \cdots & & a_{n n} & 1
\end{array}\right]\right)=\Sigma a_{i i} .
$$


$\bar{f}_{u, r, \ldots, s}(y, X)$

$$
\begin{aligned}
& =\int_{F} \cdots \int_{F^{n}} f\left(B_{u} B_{r} \cdots B_{s}\left[\begin{array}{cccc}
1 & & & \\
w_{1} & 1 & & 0 \\
\vdots & & \ddots & \\
w_{n} & 0 & \cdots & 1
\end{array}\right]\right. \\
& \cdot\left[\begin{array}{cccc}
1 & & & \\
0 & 1 & & \\
\vdots & v_{1} & \ddots & \\
0 & v_{n-1} & & 1
\end{array}\right]\left[\begin{array}{cccc}
1 & & & \\
0 & \ddots & \\
\vdots & & & \\
0 & \cdots & z_{1} & 1
\end{array}\right](y, X) \\
& \chi \chi\left(-w_{1}-v_{1}-\cdots-z_{1}\right) \frac{1}{\left|u_{1} r_{1}^{2} \cdots s_{1}^{n}\right|} d w d v \cdots d z \text {. } \\
& \int_{F} \cdots \int_{F^{n}} f\left(\left[\begin{array}{ccccc}
1 & 0 & & \cdots & 0 \\
0 & u_{1} & & \cdots & u_{n} \\
0 & 0 & r_{1} & \cdots & r_{n-1} \\
\vdots & \vdots & & \ddots & \vdots \\
0 & 0 & & \cdots & s_{1}
\end{array}\right]^{-1}\right. \\
& {\left[\begin{array}{ccccc}
1 & & & & \\
w_{1} & 1 & & & \\
w_{2} & v_{1} & 1 & & \\
\vdots & \vdots & & \ddots & \\
w_{n} & v_{n-1} & \cdots & z_{1} & 1
\end{array}\right](y, X)} \\
& \chi \chi\left(-w_{1}-v_{1}-\cdots-z_{1}\right) \frac{1}{\left|u_{1} r_{1}^{2} \cdots s_{1}^{n}\right|} d w d v \cdots d z \\
& =\int_{\Gamma_{n}} f\left(\left[\begin{array}{ccccc}
1 & 0 & \cdots & 0 \\
0 & u_{1} & \cdots & u_{n} \\
0 & 0 & r_{1} & \cdots & r_{n-1} \\
\vdots & \vdots & & \ddots & \vdots \\
0 & 0 & & \cdots & s_{1}
\end{array}\right] \gamma(y, X)\right) \phi(-\gamma) \frac{1}{\left|u_{1} r_{1}^{2} \cdots s_{1}^{n}\right|} d \gamma
\end{aligned}
$$

since Haar measure on $\Gamma_{n}$ is $d w d v \cdots d z$. 
$[\rho(b, A) f]_{u, r, \ldots, s}^{-}(y, X)$

$$
=\int_{\Gamma_{n}}[\rho(b, A) f]\left(\left[\begin{array}{ccccc}
1 & 0 & & \cdots & 0 \\
0 & u_{1} & & \cdots & u_{n} \\
0 & 0 & r_{1} & \cdots & r_{n-1} \\
\vdots & \vdots & \ddots & \vdots \\
0 & 0 & & \cdots & s_{1}
\end{array}\right] \gamma(y, X)\right)
$$

$\phi(-\gamma) \frac{1}{\left|u_{1} r_{1}^{2} \cdots s_{1}^{n}\right|} d \gamma$

$$
\begin{aligned}
= & \int_{\Gamma_{n}} f\left(\left[\begin{array}{ccccc}
1 & 0 & & \cdots & 0 \\
0 & u_{1} & & \cdots & u_{n} \\
0 & 0 & r_{1} & \cdots & r_{n-1} \\
\vdots & \vdots & \vdots & \ddots & \vdots \\
0 & 0 & & \cdots & s_{1}
\end{array}\right] \gamma(X b, \mathbf{1})(y, X A)\right. \\
& \cdot \phi(-\gamma) \frac{1}{\left|u_{1} r_{1}^{2} \cdots s_{1}^{n}\right|} d \gamma
\end{aligned}
$$

[Set $\beta=\gamma(X b, \mathbf{1})$.]

$$
\begin{aligned}
& =\int_{\Gamma_{n}} f\left(\left[\begin{array}{ccccc}
1 & 0 & & \cdots & 0 \\
0 & u_{1} & & \cdots & u_{n} \\
0 & 0 & r_{1} & \cdots & r_{n-1} \\
\vdots & \vdots & & \ddots & \vdots \\
0 & 0 & & \cdots & s_{1}
\end{array}\right] \quad \beta(y, X A)\right. \\
& \cdot \chi\left(e_{1} \cdot X b\right) \phi(-\beta) \frac{1}{\left|u_{1} r_{1}^{2} \cdots s_{1}^{n}\right|} d \beta \\
& =\chi\left(e_{1} \cdot X b\right) \bar{f}_{u, r, \ldots, s}(y, X A) \text {. }
\end{aligned}
$$

This is precisely ind ${ }_{\Gamma_{n} \uparrow G_{n}} \phi$ on $\mathcal{H}$. So we have

$$
\begin{aligned}
L^{2}\left(G_{n}\right) & \simeq \int_{F} \cdots \int_{F^{n}} \mathcal{H} d u d r \cdots d s \\
\rho_{C_{n}} & \simeq \int_{F} \cdots \int_{F^{n}}\left(\operatorname{ind}_{\Gamma_{n} \uparrow G_{n}} \phi\right) d u d r \cdots d s .
\end{aligned}
$$


Let

$$
\begin{aligned}
& \Delta_{n}=\left\{\left[\begin{array}{cccc}
u_{1} & & \cdots & u_{n} \\
0 & r_{1} & \cdots & r_{n-1} \\
\vdots & & \ddots & \vdots \\
0 & & \cdots & s_{1}
\end{array}\right]: u_{1} \neq 0, \ldots, s_{1} \neq 0\right\} \\
& =\text { group of upper triangular invertible } n \times n \text { matrices. }
\end{aligned}
$$

Right Haar measure on $\Delta_{n}$ is

$$
\frac{d u_{1} \cdots d u_{n} d r_{1} \cdots d r_{n-1} \cdots d s_{1}}{\left|u_{1} r_{1}^{2} \cdots s_{1}^{n}\right|}
$$

We may identify $\Delta_{n}$ with $\Gamma_{n} \backslash G_{n}$ as a measure space, and hence we may regard ind $\Gamma_{\Gamma_{n} \uparrow G_{n}} \phi$ as a representation $\sigma$ on $L^{2}\left(\Delta_{n}\right)$.

We now renormalize $\bar{f}_{u, r, \ldots, s}$ so that we can recapture $f$ as an integral over $\Delta_{n}$.

We have

$$
f=\int_{F} \cdots \int_{F^{n}} \bar{f}_{u, r, \ldots, s} d u d r \cdots d s
$$

Set $f_{u, r, \ldots, s}=\sqrt{\left|u_{1} r_{1}^{2} \cdots s_{1}^{n}\right|} \bar{f}_{u, r, \ldots, s} ;$ then

$$
\begin{gathered}
f=\int_{F} \cdots \int_{F^{n}} f_{u, r, \ldots, s} \frac{d u d r \cdots d s}{\left|u_{1} r_{1}^{2} \cdots s_{1}^{n}\right|}=\int_{\Delta_{n}} f_{\alpha} d \alpha \\
f_{\alpha}(y, X)=\left(\left|u_{1} r_{1}^{2} \cdots s_{1}^{n}\right|\right)^{-1 / 2} \int_{\Gamma_{n}} f\left(\alpha^{-1} \gamma(y, X)\right) \phi(-\gamma) d \gamma,
\end{gathered}
$$

where

$$
\alpha=\left[\begin{array}{ccccc}
1 & 0 & & \cdots & 0 \\
0 & u_{1} & & \cdots & u_{n} \\
0 & 0 & r_{1} & \cdots & r_{n-1} \\
\vdots & \vdots & & \ddots & \vdots \\
0 & 0 & & \cdots & s_{1}
\end{array}\right]
$$

We thus have $L^{2}\left(G_{n}\right) \simeq \int_{\Delta_{n}} L^{2}\left(\Delta_{n}\right) d \alpha, \rho_{G_{n}} \simeq \int_{\Delta_{n}} \sigma d \alpha$. We may identify $\int_{\Delta_{n}} L^{2}\left(\Delta_{n}\right) d \alpha$ with $L^{2}\left(\Delta_{n}\right) \otimes L^{2}\left(\Delta_{n}\right), \rho_{G_{n}} \simeq \sigma \otimes \mathbb{1}$. 


\section{REFERENCES}

[1] L. Auslander and C. Moore, Unitary representations of solvable Lie groups, Amer. Math. Soc. Memoir no. 62, 1966.

[2] B. Blackadar, The regular representation of restricted direct product groups, J. Funct. Anal., 25 (1977), 267-274.

[3] N. Jacobson, Basic Algebra II, W. H. Freeman, San Francisco, 1980.

[4] H. Jacquet, Generic Representations, Lecture Notes in Mathematics v. 587, SpringerVerlag, 1977, 91-101.

Received March 21, 1981.

UNIVERSITY OF NEVADA, RENO

RENO, NV 89557 


\title{
PACIFIC JOURNAL OF MATHEMATICS EDITORS
}

\author{
Donald BabBitT (Managing Editor) \\ University of California \\ Los Angeles, CA 90024 \\ Hugo Rossi \\ University of Utah \\ Salt Lake City, UT 84112 \\ C. C. Moore and Arthur Ogus \\ University of California \\ Berkeley, CA 94720
}

\author{
J. DugundiI \\ Department of Mathematics \\ University of Southern California \\ Los Angeles, CA 90089-1113 \\ R. FINN and H. SAMELSON \\ Stanford University \\ Stanford, CA 94305
}

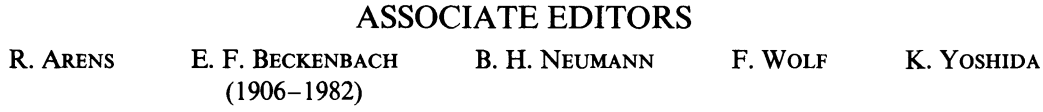

\section{SUPPORTING INSTITUTIONS}

UNIVERSITY OF ARIZONA
UNIVERSITY OF BRITISH COLUMBIA
CALIFORNIA INSTITUTE OF TECHNOLOGY
UNIVERSITY OF CALIFORNIA
MONTANA STATE UNIVERSITY
UNIVERSITY OF NEVADA, RENO
NEW MEXICO STATE UNIVERSITY
OREGON STATE UNIVERSITY

\author{
UNIVERSITY OF OREGON \\ UNIVERSITY OF SOUTHERN CALIFORNIA \\ STANFORD UNIVERSITY \\ UNIVERSITY OF HAWAII \\ UNIVERSITY OF TOKYO \\ UNIVERSITY OF UTAH \\ WASHINGTON STATE UNIVERSITY \\ UNIVERSITY OF WASHINGTON
}

The Supporting Institutions listed above contribute to the cost of publication of this Journal, but they are not owners or publishers and have no responsibility for its content or policies.

Mathematical papers intended for publication in the Pacific Journal of Mathematics should be in typed form or offset-reproduced (not dittoed), double spaced with large margins. Please do not use built up fractions in the text of the manuscript. However, you may use them in the displayed equations. Underline Greek letters in red, German in green, and script in blue. The first paragraph must be capable of being used separately as a synopsis of the entire paper. In particular it should contain no bibliographic references. Please propose a heading for the odd numbered pages of less than 35 characters. Manuscripts, in triplicate, may be sent to any one of the editors. Please classify according to the scheme of Math. Reviews, Index to Vol. 39. Supply name and address of author to whom proofs should be sent. All other communications should be addressed to the managing editor, or Elaine Barth, University of California, Los Angeles, California 90024.

There are page-charges associated with articles appearing in the Pacific Journal of Mathematics. These charges are expected to be paid by the author's University, Government Agency or Company. If the author or authors do not have access to such Institutional support these charges are waived. Single authors will receive 50 free reprints; joint authors will receive a total of 100 free reprints. Additional copies may be obtained at cost in multiples of 50 .

The Pacific Journal of Mathematics is issued monthly as of January 1966. Regular subscription rate: $\$ 132.00$ a year (6 Vol., 12 issues). Special rate: $\$ 66.00$ a year to individual members of supporting institutions.

Subscriptions, orders for numbers issued in the last three calendar years, and changes of address should be sent to Pacific Journal of Mathematics, P.O. Box 969, Carmel Valley, CA 93924, U.S.A. Old back numbers obtainable from Kraus Periodicals Co., Route 100, Millwood, NY 10546.

The Pacific Journal of Mathematics ISSN 0030-8730 is published monthly by the Pacific Journal of Mathematics at P.O. Box 969, Carmel Valley, CA 93924. Application to mail at Second-class postage rates is pending at Carmel Valley, California, and additional mailing offices. Postmaster: Send address changes to Pacific Journal of Mathematics, P. O. Box 969, Carmel Valley, CA 93924.

PUBLISHED BY PACIFIC JOURNAL OF MATHEMATICS, A NON-PROFIT CORPORATION

Copyright $(1983$ by Pacific Journal of Mathematics 


\section{Pacific Journal of Mathematics}

Vol. 108, No. $2 \quad$ April, 1983

Enrique Atencia and Francisco Javier Martin-Reyes, The maximal ergodic Hilbert transform with weights $\ldots \ldots \ldots \ldots \ldots \ldots \ldots \ldots \ldots . \ldots 257$

Bruce Blackadar, The regular representation of local affine motion

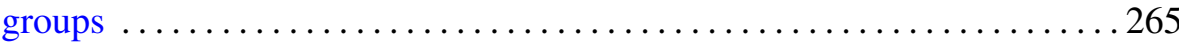

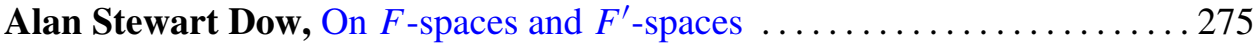

Yoshifumi Kato, On the vector fields on an algebraic homogeneous space . . 285

Dmitry Khavinson, Factorization theorems for different classes of analytic functions in multiply connected domains $\ldots \ldots \ldots \ldots \ldots \ldots \ldots \ldots 295$

Wei-Eihn Kuan, A note on primary powers of a prime ideal . . . . . . . . 319

Benjamin Michael Mann and Edward Yarnell Miller, Characteristic classes for spherical fibrations with fibre-preserving free group

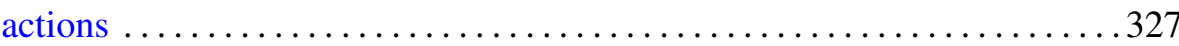

Steven Alan Pax, Appropriate cross-sectionally simple four-cells are flat . . . 379 R. K. Rai, On orthogonal completion of reduced rings ................ 385

V. Sree Hari Rao, On random solutions of Volterra-Fredholm integral

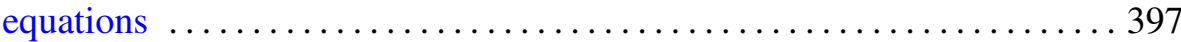

Takeyoshi Satō, Integral comparison theorems for relative Hardy spaces of solutions of the equations $\Delta u=P u$ on a Riemann surface $\ldots \ldots \ldots . .407$

Paul Sydney Selick, A reformulation of the Arf invariant one $\bmod p$ problem and applications to atomic spaces

Roelof Jacobus Stroeker, Reduction of elliptic curves over imaginary quadratic number fields

Jacob Towber, Natural transformations of tensor-products of representation-functors. I. Combinatorial preliminaries

James Chin-Sze Wong and Abdolhamid Riazi, Characterisations of amenable locally compact semigroups 